\title{
Endoscopic Ultrasound-Guided Drainage of a Biloma: A Novel Approach
}

A 42-year-old man with symptoms of cholecystitis underwent open cholecystectomy. The gallbladder was found to be distended with pus and the dilated cystic duct contained a stone. The common bile duct was not explored. He was referred to our hospital 30 days later with a 1-week history of fever and progressive jaundice, associated with pain and tenderness over the right upper quadrant of his abdomen. Computed tomography of his abdomen confirmed that there was a fluid collection in the gallbladder fossa (Figure 1), with a mildly dilated common bile duct.

An endoscopic retrograde cholangiogram performed the following day revealed a bile leak from the cystic duct stump and a stone in the proximal common bile duct. Sphincterotomy was performed and the stone was removed using a Dormia basket. A 10-cm-long 10-Fr plastic biliary endoprosthesis (Wilson-Cook Medical Inc., Winston-Salem, North Carolina, USA) was inserted. Using a linear echo endoscope (Olympus UCP 160; Olympus Co., Tokyo, Japan), a $6 \mathrm{~cm} \times 5 \mathrm{~cm}$ fluid collection was visualized in the gallbladder fossa. A 19-guage Wilson-Cook needle was introduced and $180 \mathrm{ml}$ of bile was aspirated, emptying the collection (Figure 2 ). The patient made a complete symptomatic recovery, with resolution of his fever and jaundice and improvement in his biochemical markers.

A repeat endoscopic ultrasound was performed 2 weeks later which confirmed complete resolution of the fluid collection. He was electively readmitted 6 weeks later for stent removal, and cholangiography at that time revealed no evidence of a further bile leak. Two months later he had a transabdominal ultrasound examination, which confirmed the resolution of his biloma, and he remains well.

Endoscopic ultrasound-guided fine-needle aspiration of the infected biloma, together with endoscopic biliary stent placement, resulted in complete resolution of this patient's biloma, with no fur-

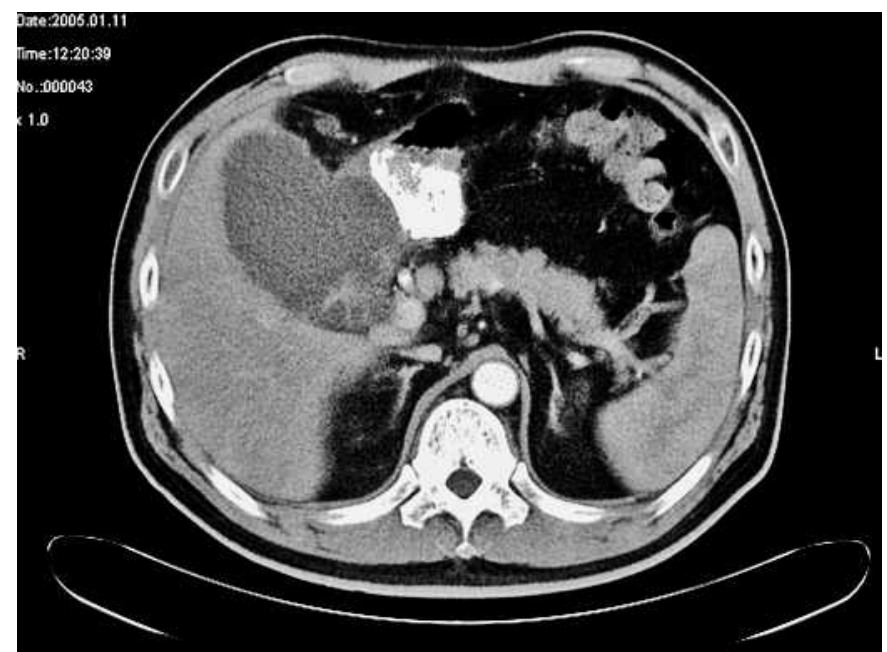

Figure 1 Computed tomographic image showing a large collection of fluid in the gallbladder fossa.

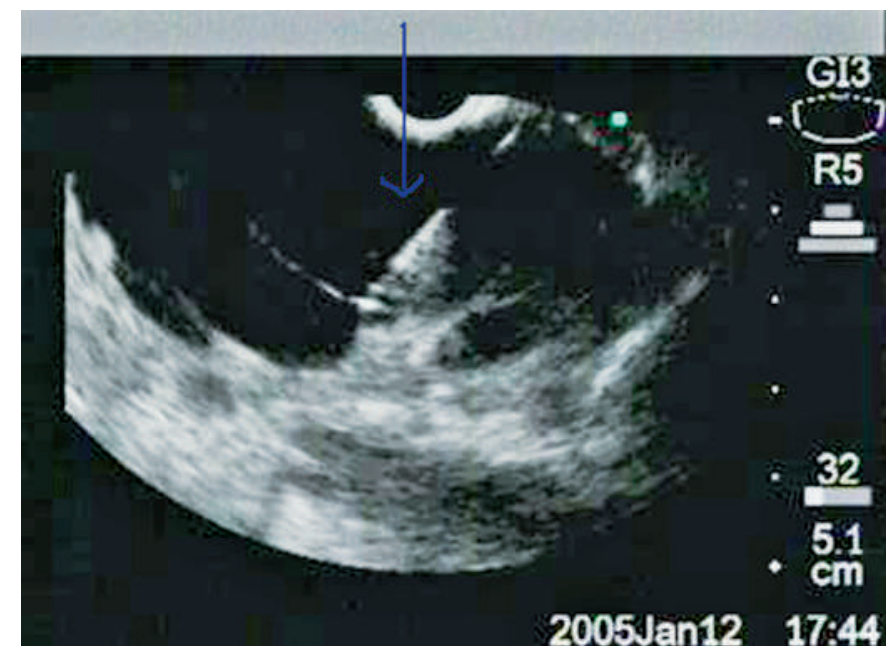

Figure 2 Endoscopic ultrasound image showing the 19-guage needle within the biloma.

ther reaccumulation of bile in the gallbladder fossa. This was a safe, simple, and effective procedure that was used to treat a patient with a potentially complex problem.

\section{Competing interests: None}

Corresponding Author

\section{R. Ponnudurai, M.D.}

Hepatology Unit

Selayang Hospital

Lebuhraya Selayang Kepong

Batu Caves

Selangor 68100

Malaysia

Fax: $\quad+60-361207564$

E-mail: ryanmd66@hotmail.com 